\title{
«À la recherche des Macédoniens » : le regard des cartes, 1840-1918
}

Joëlle Dalègre

\section{OpenEdition}

1 Journals

Édition électronique

URL : https://journals.openedition.org/ceb/782

DOI : $10.4000 /$ ceb.782

ISSN : 2261-4184

Éditeur

INALCO

\section{Édition imprimée}

Date de publication : 30 mars 2011

Pagination : 115-126

ISBN : 978-2-85831-189-7

ISSN : 0290-7402

\section{Référence électronique}

Joëlle Dalègre, « «À la recherche des Macédoniens » : le regard des cartes, 1840-1918 », Cahiers

balkaniques [En ligne], 38-39 | 2011, mis en ligne le 02 décembre 2011, consulté le 06 juillet 2021. URL http://journals.openedition.org/ceb/782 ; DOl : https://doi.org/10.4000/ceb.782

Ce document a été généré automatiquement le 6 juillet 2021.

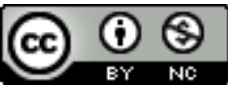

Cahiers balkaniques est mis à disposition selon les termes de la Licence Creative Commons Attribution - Pas d'Utilisation Commerciale 4.0 International. 


\title{
«À la recherche des Macédoniens » : le regard des cartes, $1840-1918$
}

\author{
Joëlle Dalègre
}

\section{Yugoslavia, Macedonia, Balkans}

1 L'expression « péninsule balkanique » a été employée pour la première fois en 1808 par l'Allemand August Zeune, en 1821, un autre Allemand, d'Oetzel, dessina la première carte des populations européennes. Dès lors les cartes, qu'elles soient dites ethnographiques, ethnologiques, ethnolinguistiques, ethnocratiques... se sont multipliées jusqu'en 1920. Peu à peu se sont précisées les techniques comme la connaissance des régions et des populations concernées, et l'intérêt s'est focalisé sur le sud-est de l'Europe.

2 Après avoir rappelé quelques traits communs à toutes ces cartes, je vais montrer, par quelques exemples, comment a évolué la dénomination des populations habitant la Macédoine ottomane, jusqu'à découvrir au début du XXe siècle, la notion de « Macédoslaves ".

3 En 80 ans, 1842-1920, une centaine de cartes ont concerné, de loin, puis de plus près, la Macédoine au sens géographique du terme. Ce nombre reflète à la fois l'intérêt et l'emprise de l'Europe occidentale sur la région. Les cartes sont toutes tracées par des ressortissants des grandes puissances européennes, sauf trois exceptions au début du $\mathrm{XX}^{\mathrm{e}}$ siècle, le Serbe Jovan Cvijic, le Bulgare Ivanov et le Grec Sotériadès. Ces cartes sont publiées le plus souvent en Grande-Bretagne chez Stanford ou en Allemagne, à Gotha, chez Julius Perthes, pour satisfaire la curiosité d'un public étranger à la région. Signe des temps, ces puissances s'intéressent pour la première fois aux populations, et pas seulement aux territoires, même si leurs décisions finales et le tracé des frontières ont peu tenu compte de ces considérations humaines. Peu à peu les cartes se précisent. Techniquement le tracé des éléments naturels est plus exact, on passe de noms largement étalés en travers d'un espace non délimité (cartes d'Oetzel, de Müller en 1842 ou de Kombst en 1843), à des surfaces à plat monochromes, pour enfin, à partir de 
Kiepert en 1876, adopter des bandes de hachures intégrées à la couleur dominante. C'est dire que l'on a pris conscience de l'imbrication des populations.

4 La carte passe pour une représentation du réel, pour une preuve scientifique, on en multiplie donc le nombre dans les périodes critiques, 27 cartes entre 1890 et 1912 pendant la préparation des guerres balkaniques, 31 entre la fin de ces guerres et 1919. Une fois les frontières tracées, au contraire, les cartes ethnologiques se font rares, car aucun des États des Balkans ne tient à avouer qu'il pourrait exister chez lui d'importantes "minorités ». La carte est une arme diplomatique, destinée à prouver aux États décideurs la justesse des revendications des uns ou des autres, aussi sa publication est-elle souvent financée par un gouvernement qui, parfois, fournit même les données utilisées. On peut ainsi citer la carte signée Sotériadès, professeur de l'Université d'Athènes, publiée chez Stanford en 1918, qui, en réalité, est l'œuvre d'un colonel de l'armée grecque, Katsikoyannis, mandaté par Venizélos, le premier ministre. Une autre anecdote est fort connue : le géographe allemand Kiepert, Grand Prix de Cartographie à l'Exposition universelle de 1877, publie en 1876 une carte ethnologique qui sert de référence à tous les diplomates. Les Grecs cependant s'estiment lésés, aussi la Société pour la propagande des Lettres Grecques dépêche-t-elle l'historien Paparrigopoulos, à Berlin, une célébrité dans toute l'Europe d'alors, pour plaider leur cause et obtenir un tracé plus favorable. Kiepert ne se dédie pas, mais, en 1878, année du Congrès de Berlin, il imagine, pour " $M$. Zaphiropoulos de Marseille ", une carte dite " ethnocratique ", terme inventé pour l'occasion; elle dessine des États qui, en plus des facteurs ethniques, tiennent compte des facteurs culturels, et, par ce biais, repousse la frontière grecque, en s'inspirant de Byzance, très au nord, loin des côtes égéennes.

Indépendamment des pressions diplomatiques évidentes ou des progrès techniques effectués, le contenu de la carte varie avec la nationalité de son concepteur, l'origine de ses données et la définition des termes qu'il emploie. Les signataires sont le plus souvent des militaires, parfois des voyageurs, des agents diplomatiques ou enfin, des universitaires; rares sont ceux sauf Ami Boué, Lejean ou Cvijic qui aient une réelle expérience de la région qu'il reste d'ailleurs difficile d'inventorier dans sa totalité, village par village; de plus, les voyageurs sont souvent dépendants de leur interprète. Si certaines cartes, classées par groupes comme "slaves » ou "grecques ", avantagent outrageusement, sans vergogne aucune, leur groupe de référence en niant parfois l'évidence, la manipulation des dénominations est le premier facteur de différence. Les chiffres utilisés sont, eux, souvent les mêmes, tirés des recensements ottomans qui ne distinguent pas les langues, mais les religions, ou les données fournies par chacune des communautés. En revanche, si l'on regroupe tous les musulmans comme "Turcs », on leur donne alors une importance écrasante. Mais en Macédoine ou en Thrace, indiquer les Pomaks comme "Turcs» parce que musulmans, ou "Bulgares" parce que bulgarophones, ou " Pomaks " parce que bulgarophones, mais non orthodoxes, change tout. De la même manière, indiquer tous les orthodoxes comme "Grecs", ou même tous les exarchistes, après 1870 , comme "Bulgares » change totalement le résultat. Tenter la démarcation entre les langues slaves, à un moment où le paysan est souvent incapable de donner un nom à la langue qu'il parle, est aussi une opération aux conséquences importantes, particulièrement dans l'espace macédonien. Et que faire des Valaques ou des musulmans hellénophones? Avoir une langue et une religion suffit-il à définir une conscience nationale? Certains tentent de subtiles distinctions, ainsi le consul autrichien à Andrinople, Sax, fait-il figurer 28 types de populations différentes sur sa carte en combinant langue, religion et sentiment national; " la langue n'est 
qu'un des signes divers de la nationalité. En Orient la religion et la conscience nationale ont autant d'importance pour déterminer la nationalité : il faut combiner ces trois éléments » a-t-il écrit ${ }^{1}$. De même, en 1887, Kariç, soucieux de différences, indique des « Albanais chrétiens d'origine serbe » et des « Albanais musulmans d'origine serbe »...

Les cartographes, prenant conscience progressivement de la diversité des populations, affinent peu à peu leurs dénominations, c'est ainsi qu'au début $\mathrm{du} \mathrm{XX}^{\mathrm{e}}$ siècle, dans un ensemble jusque-là désigné comme "Bulgare», certains inscrivent des «Macédoslaves ». De cette progression, je fournis ci-dessous quelques exemples.

Il s'agit de reproductions des schémas simplifiés publiés par Wilkinson dans son excellente étude sur la Macédoine ${ }^{2}$. Les originaux se trouvent tous à la B.N., section des cartes, mais la complexité des couleurs en rend lecture et reproduction difficiles, le Britannique a simplifié les données et en a extrait le seul espace macédonien.

ex 1. L'anthropologue Allemand Kombst, en 1843, a fait figurer dans un Atlas cette carte qui, dans la lignée de celles d'Oetzel en 1821 et de Müller en 1842, ne délimite aucune surface et se contente de noms vagues largement étalés ; chez d'Oetzel on rencontrait des « Illyriens », des « Turcs » et des « Grecs », chez Müller, des « Pélasges », des «Zingares », des « Illyriens » et des « Albanais ». Chez Kombst viennent s'ajouter des «Souliotes » et des « Ruméliotes » tandis que « Serbes » et « Bulgares » sont repoussés nettement au nord de l'espace macédonien.

\section{G. Kombst, 1843, Ethnographic map of Europe}

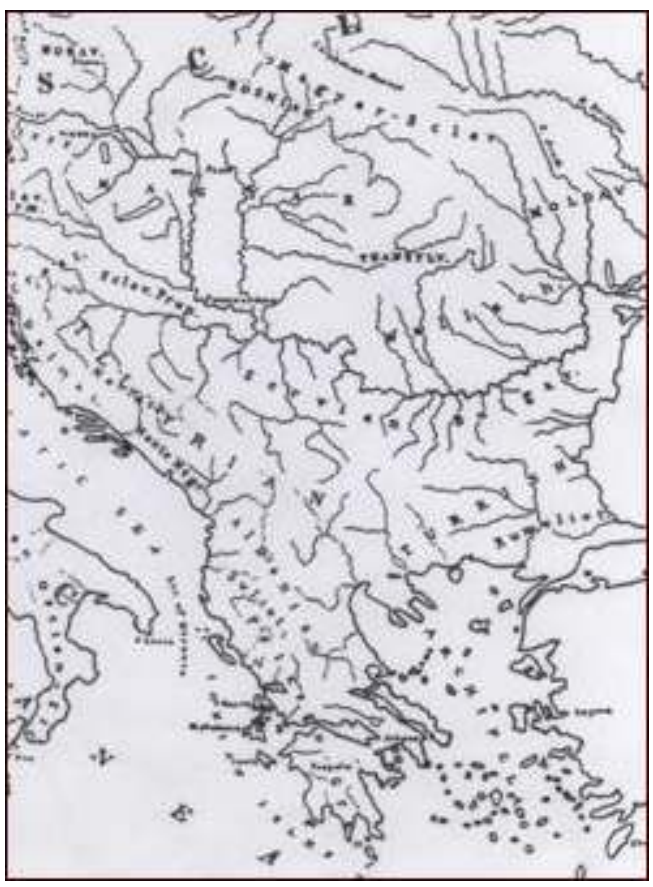

(Wilkinson, op. cit., fig. 7, p. 25, échelle inconnue)

ex 2. Lejean appartient au groupe des diplomates et voyageurs ${ }^{3}$, il a voyagé dans plusieurs continents et, au milieu du XIX ${ }^{e}$ siècle, il est vice-consul et voyage dans la Turquie d'Europe pour le compte du ministère français des Affaires étrangères. Il emploie des dénominations plus « modernes », Grecs, Turcs, Bulgares, Serbes, Albanais, Valaques. La ligne Salonique-Kavalla marque pour lui la limite nord du secteur qu'il juge grec, tandis qu'à l'ouest de Monastir (Bitola) figurent quelques noyaux serbes au 
contact de populations albanaises. Il insiste sur le fait que la présence grecque se limite à des pêcheurs et des marins sur toute la longueur des côtes ; l'État grec cependant, encore très éloigné de la Macédoine, n'émet aucune protestation à l'époque de la parution de cette carte, mais, 40 ans plus tard, ses représentants font courir le bruit que Lejean était vendu aux Bulgares! En effet, il leur attribue les 4/5e de la Macédoine géographique, y compris les villes de Kastoria et d'Ochrid. Cette carte fait largement autorité dans le demi-siècle suivant, la Grande Bulgarie dessinée à San Stefano reprend dans ses grandes lignes la limite des Bulgares selon la carte de Lejean qui sert également de base à Kiepert (qui la modifie en tenant compte de quelques compléments fournis par des Grecs).

\section{G. Lejean, 1861, Carte ethnologique de la Turquie d'Europe et des États vassaux autonomes}

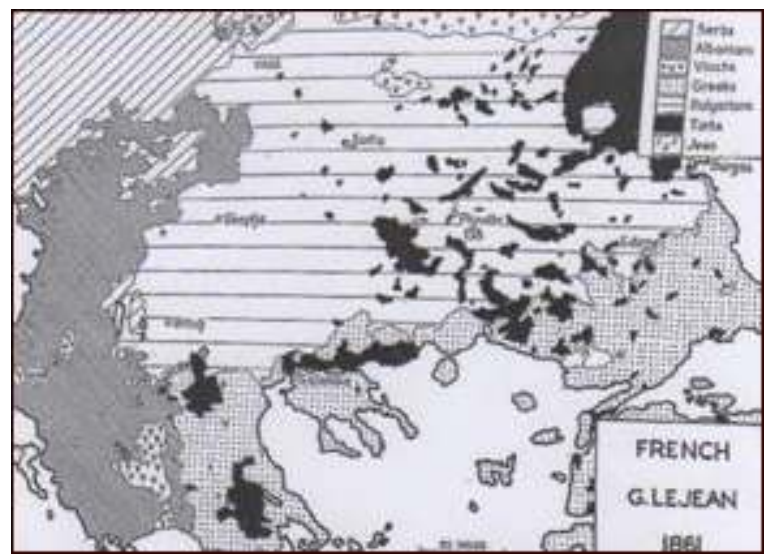

(Wilkinson, op. cit., détail fig. 11, p. 44)

ex 3. Les deux cartes de Kiepert. La carte de 1876 est la première à utiliser le système des bandes alternées sur la même surface, elle n'est guère différente de celle de Lejean, tout en accordant néanmoins un peu plus de surface aux Grecs vers le nord, puisqu'elle leur attribue la ville d'Edessa (Vodena) et une large part de l'Épire retirée ainsi aux Albanais; elle limite les enclaves valaques dans le Pinde et élimine les Serbes de l'Ouest macédonien. Avec la carte de Lejean, elle est utilisée lors des négociations du Congrès de Berlin pour soutenir les demandes bulgares. En revanche, le schéma de commande, dit ethnocratique, de 1878 contredit totalement les cartes précédentes en mettant en avant des raisons « culturelles »; ainsi une très large part de l'Albanie et de la zone macédonienne irait aux Grecs. 
3. H. Kiepert, 1876, Ethnographische Karte Übersichtkarte des Europäischen Orient

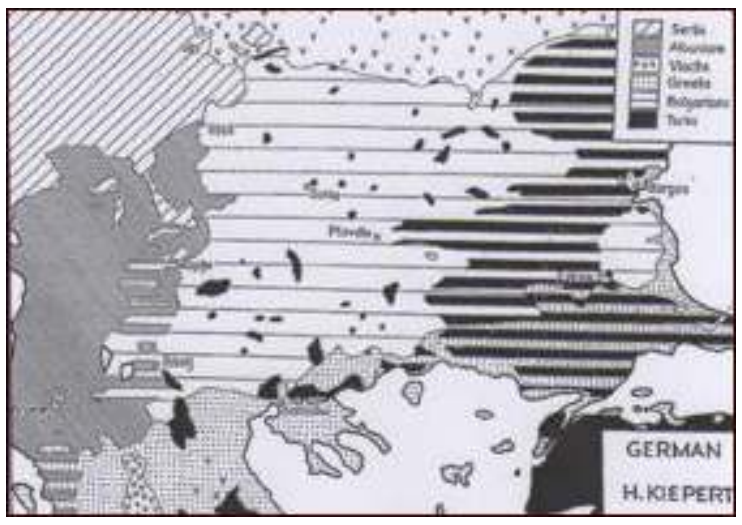

(Wilkinson, op. cit., détail fig. 15, p. 66)

3b. H. Kiepert, 1878, Ethnokratische Karte

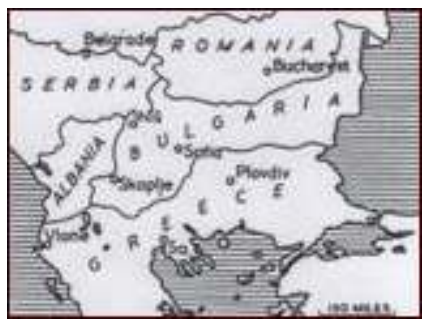

(Wilkinson, op. cit., fig. 16, p. 68)

ex 4. Sax : la première carte qui combine religion et langue, ce qui multiplie les subdivisions (28 au total, ici regroupées), la première à individualiser les « Pomaks », la première à tenter d'évaluer (on ignore par quels moyens) le sentiment national des populations en signalant des locuteurs du valaque, du bulgare ou de l'albanais « au cœur grec ", c'est enfin la première à indiquer une telle diversité de populations en Macédoine, sans majorité écrasante, avec une telle précision de détail qui suppose un excellent réseau local de renseignements auprès du consulat autrichien. 
K. Sax, 1877, Ethnographische Karte des Europäischen Türkei und ihrer Dependenzen zu Anfang des Jahres 1877

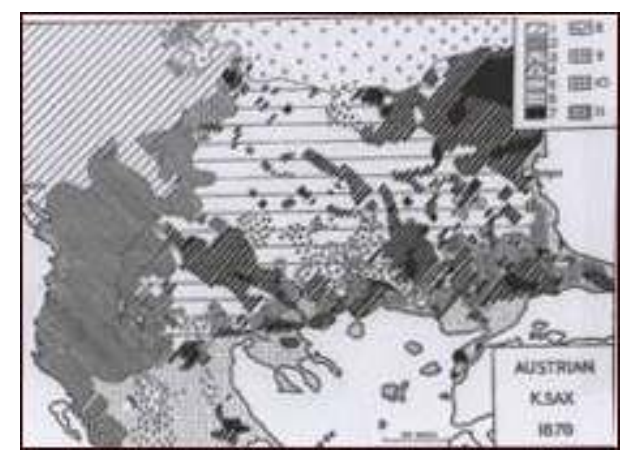

1. Serbo-Croates (divisés en trois groupes religieux)

2. Albanais (divisés en trois groupes religieux)

3. Roumains

4. Bulgares Pomaks

5. Grecs

6. Bulgares exarchistes

7. Tatars, Circassiens, Turcs, Turcomans

8. Gréco-Valaques

9. Serbo-Bulgares

10. Gréco-Bulgares

11. Gréco-Albanais

ex 5, 6 et 7, les cartes de Cvijić, Stanford et Sotériadès.

8 Ces trois cartes indiquent ce qu'elles nomment des "macédoslaves ", là où jusqu'alors voyageurs et cartographes n'indiquaient que des «Bulgares ». Il s'agit de la carte du Serbe Cvijic en 1909, de celle publiée en Grande Bretagne par les éditions Stanford qui reprend les données de Civjic auxquelles s'ajoutent quelques éléments grecs, en 1917, et, plus exceptionnelle encore (vue depuis notre époque) celle du Grec Sotériadès en 1918, publiée à l'instigation de Venizélos et qui indique à l'intérieur même des frontières grecques issues du traité de 1913, des "macédoslaves ». C'est l'affirmation d'une nouvelle composante dans la mosaïque ethnolinguistique de la Macédoine. 


\section{J. Cvijić, 1909, Carte ethnographique de la péninsule des Balkans}

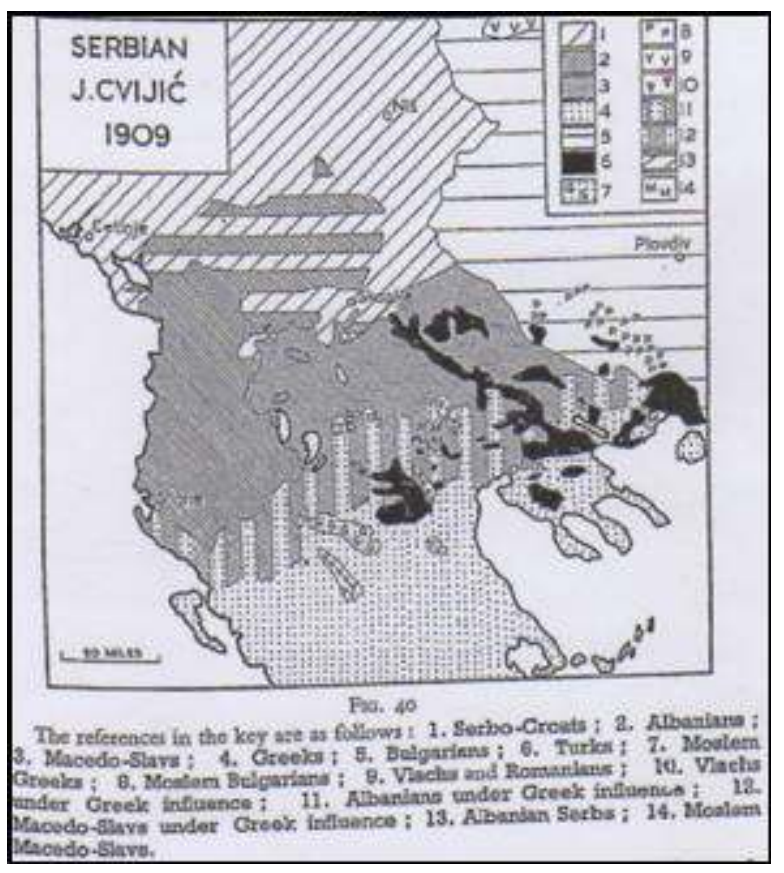

(Wilkinson, op. cit., détail fig. 40, p. 163)

6. E. Stanford, 1917, A sketch map of the linguistic areas of Europe

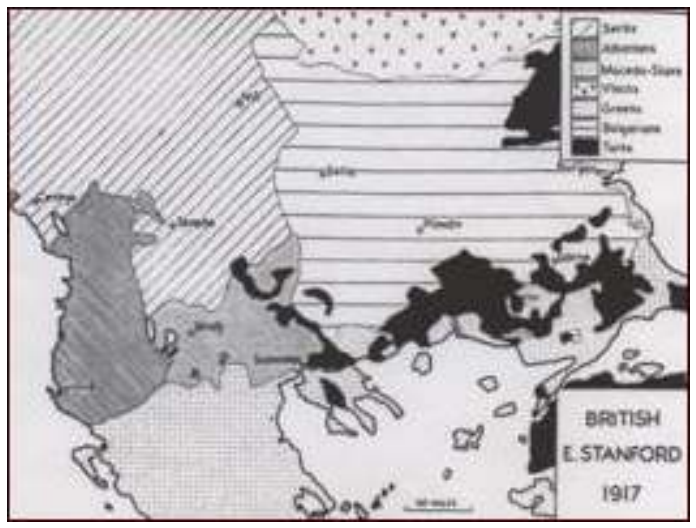

(Wilkinson, op. cit., détail fig. 53, p. 220)

Zaryansko, un Russe, dans sa carte de 1890, avait laissé, entre Serbes et Bulgares, une zone blanche portant le mot "slave", avouant ainsi son impuissance à préciser. Peucker, en 1903, signale dans le commentaire de sa carte "slave » que les Slaves de Macédoine sont différents des autres car porteurs d'une culture byzantine, mais il ne les indique pas séparément sur sa carte. L'année 1903 est aussi celle où Misirkov, un Macédonien (né à Pella aujourd'hui en Grèce) vivant en Russie, dans un livre «Des affaires macédoniennes", parle le premier d'une langue macédonienne, de la nécessité d'en faire une langue littéraire en prenant pour base les dialectes de la région VelesPrilep-Bitola-Ochrid; il affirme l'existence d'une ethnie macédonienne différente des Serbes ou à des Bulgares, ce que reprendra l'année suivante le plaidoyer Pro Macedonia de Victor Bérard qui élimine également les prétentions grecques. C'est le volet littéraire et scientifique d'une affirmation politique qui croît depuis la fin du XIX ${ }^{\mathrm{e}}$ siècle 
avec l'ORIM et ses divisions entre acceptation ou refus de la mainmise bulgare sur les «Macédoniens ».

\section{G. Sotériades, 1918, Hellenism in the Near East, an ethnological map}

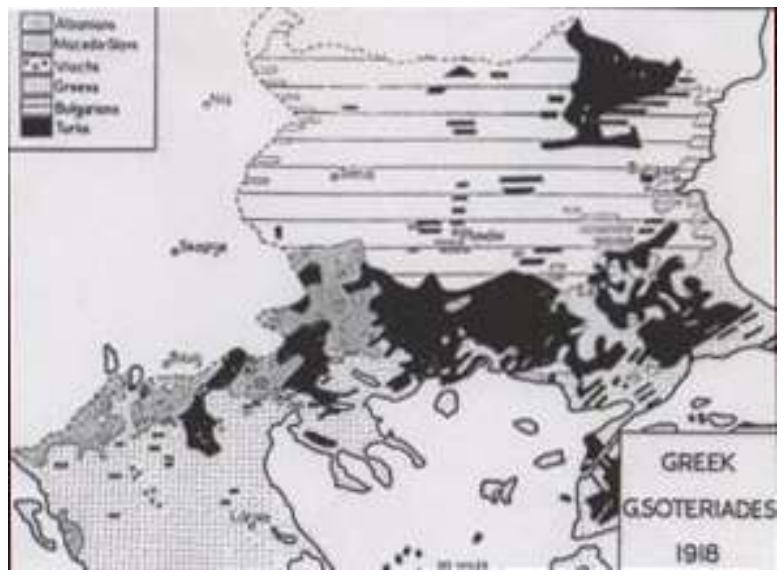

(Wilkinson, op. cit., détail fig. 45, p. 190)

10 L'affirmation cartographique du «macédoslave» vient avec les travaux du Serbe Cvijic; il est chef du département géographique de l'Université de Belgrade, professeur invité à la Sorbonne pendant la Première Guerre mondiale, puis responsable de la délégation serbe à la Conférence de la Paix en 1918, sa parole fait autorité. Dans sa Géographie humaine, il introduit le mot "macédoslave " en 1906 et consacre l'année suivante un article entier à ce sujet. Il affirme que Skopje ou Tetovo ne sont pas la "Macédoine », mais la "Vieille Serbie », que la masse des Slaves de Macédoine ne se sent ni serbe, ni bulgare et il démonte les mécanismes employés par les linguistes, les historiens, les folkloristes et les cartographes pour les assimiler aux uns ou aux autres. La langue macédonienne, écrit-il, est une "transition entre le bulgare et le serbo-croate 4 " et n'empêche pas la compréhension des deux autres langues; la population a été fortement influencée par la culture byzantine, mais cela n'a pas créé de conscience nationale, le nom de Bulgare qui leur est donné par l'ensemble des voyageurs et des cartographes est une erreur due à leur dépendance face aux traducteurs et à la confusion faite entre « Bulgare » et « paysan » quand il s'agit de désigner des personnes qui ne sont ni Grecques ni musulmanes. Les Grecs, dit-il à raison, traitent n'importe quel slavophone de « Bulgare ».

11 Ces personnes, écrit-il, n'ont pas encore de conscience nationale nette, et se définissent selon les circonstances locales et au gré de leurs intérêts : «Il est impossible de voir dans la masse de la population slave macédonienne des Serbes de race ou des Bulgares de race. Tout au plus pourrait-on distinguer parmi les Slaves macédoniens ceux du parti bulgare, ceux du parti serbe et ceux du parti grec, formés par la propagande et par d'autres influences. Ces partisans ne constituent d'ailleurs qu'une petite fraction de la population slave... on trouverait ainsi dans la même famille des Serbes, des Bulgares et des Grecs $!^{5} \mathrm{Et}$ il en conclut qu'ils seront très facilement assimilés en une génération aux Serbes ou aux Bulgares selon les décisions politiques futures.

On voit sur sa carte qu'il évacue quasi totalement les Bulgares de l'espace macédonien, attribuant le nord de la région aux Serbes, le centre aux « macédoslaves » et le sud aux " macédoslaves sous influence grecque ». On retrouve cette répartition, simplifiée, sur 
la carte de Stanford en 1917 ; la carte de Sotériadès accepte même au nord-ouest de la Grèce la présence de ces « macédoslaves » et insiste sur la présence turque à l'est.

«Nulle part en Europe les nationalités ne sont aussi mêlées que dans la Macédoine, particulièrement dans sa partie méridionale. Il y a en outre des transitions d'une nationalité à une autre, principalement une fusion de Slaves avec les Grecs, les Turcs et les Albanais, des Tsintsares avec les Grecs et les Slaves. Cette fusion est plus ou moins complète : en certains endroits, elle ne fait que commencer, en d'autres, elle est déjà bien avancée, ailleurs elle est presque achevée. Enfin, la masse du peuple chez les Slaves macédoniens n'a pas une conscience nationale bien nette; elle n'a pas de passé historique qui lui appartienne en propre, elle n'a pas de langue littéraire. Elle parle une langue que les uns considèrent comme très voisine $d u$ serbe, d'autres du bulgare, que d'autres encore prétendent être une langue slave spéciale. Il n'a donc pas de signes ethnographiques assez sûrs pour permettre de désigner comme Serbes ou comme Bulgares les masses de la population slave ${ }^{6}$.

On retrouve ces «Macédoslaves » sur plusieurs autres cartes éditées en GrandeBretagne et inspirées de celles de Cvijic, sur deux cartes grecques de 1914 et 1918 (celles de Nikolaïdis et Sotériadès). Serbes et Grecs sont d'accord pour préférer voir des « Macédoslaves » plutôt que des « Bulgares »). Signalons enfin, à titre anecdotique, une carte des Affaires étrangères allemandes de 1940 qui distingue des « Macédoniens aux affirmations bulgares » des «Macédoniens aux affirmations serbes" et des « Macédoniens douteux »!

En conclusion pour relativiser les données de cette étude, je veux citer Nicholson, secrétaire du président Wilson à la Conférence de la Paix ${ }^{7}$ :

"Chacune des nationalités de l'Europe centrale apporte une cargaison de statistiques et de cartes géographiques truquées. Quand les statistiques échouent, on sort les cartes en couleurs. Un gros volume ne suffirait pas à analyser les différents types de cartes maquillées que la guerre et la Conférence de la Paix ont fait naître. Un nouvel instrument politique, le langage des cartes, a été inventé. Une carte vaut autant qu'une bonne affiche, mais étant une carte, elle prend une apparence respectable et authentique. Une bonne carte est une bouée de sauvetage pour maint argument défaillant et coulant à pic. C'est surtout dans les Balkans que ce procédé atteint son apogée ».

\section{NOTES}

1. Jovan Cvijic, Remarques sur l'ethnographie de la Macédoine, Paris, Roustan, 1907, p. 37.

2. Henry R. Wilkinson, Maps and politics, a review of cartography of Macedonia, Liverpool Studies in Geography University Press, 1951.

3. M.T.Lorrain, Guillaume Lejean : voyageur et géographe (1824-1871), Perséides, 2006, 344 p.

4. Jovan Cvijic, op. cit., p. 16.

5. Jovan Cvijic, Ibidem, p. 46.

6. Jovan Cvijic, Ibidem, p. 15.

7. Cité dans Dimitris Kitsikis, Propagande et pressions en politique internationale: la Grèce à la Conférence de la Paix, 1919-1920, Paris, P.U.F, 1963, p. 169. 


\section{RÉSUMÉS}

Comment peu à peu les cartes ethnographiques découvrent et signalent, en Macédoine, à l'aube $\mathrm{du} \mathrm{XX}^{\mathrm{e}}$ siècle, des « Macédoniens slaves »

L'expression "péninsule balkanique » est employée pour la première fois en 1808 par August Zeune, en 1821, un autre Allemand, d'Oetzel, dessine la première carte des populations européennes. Les cartes se multiplient jusqu'en 1920. Peu à peu se précisent les techniques, la connaissance des régions et des populations; les cartes deviennent une arme de propagande, attribuant, selon le concepteur, le territoire macédonien aux Grecs, aux Serbes et majoritairement aux Bulgares. Le Serbe Cvijic, en 1906, le premier, situe sur une carte, des «macédoslaves». Cette communication présente les étapes principales de cette évolution cartographique qui aboutit à découvrir des « Macédoniens » en... Macédoine.

The expression 'Balkan peninsula' was first employed in 1808 by August Zeune. In 1821 another German, d'Oetzel, drew the first map of the European populations. The maps continued multiplying until 1920. Little by little the techniques as well as the knowledge of the various regions and populations became more precise. The maps became a propaganda tool, in as much as they attributed-depending on the cartographer-Macedonian territory to the Greeks, the Serbs or, most often, the Bulgarians. In 1906, the Serb Cvijic, the first, situated on a map, the "macédoslaves". This communication outlines the principal stages of this cartographic evolution, which eventually situated the "Macedonians" in none other than... Macedonia.

\section{INDEX}

Index chronologique : dix-neuvième siècle, vingtième siècle

Keywords : macedonian, Cvijic Jovan (1865-1927), ethnological map, Slavomacedonian, Balkans, Yugoslavia, Monastir, Greece, Edessa, nineteenth century, History

motsclesmk ЈУГОСЛАВИЈА, БАЛКАНОТ, МАКЕДОНИЈА, ОХРИД, БИТОЛА

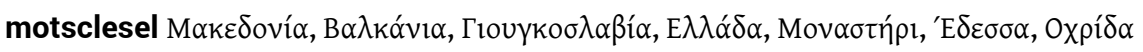

Index géographique : Macédoine, Balkans, Yougoslavie, Grèce, Monastir, Edessa, Ohrid

Thèmes : Histoire

Mots-clés : Cvijić Jovan (1865-1927), Macédoniens, Cvijić Jovan (1865-1927), cartes

ethnographiques, Slavomacédoniens

motsclestr Makedonya, Balkanlar, Yugoslavya, Yunanistan, Monastir, Ohrid, Edhessa, Bitola

\section{AUTEUR}

JOËLLE DALÈGRE

MCF HDR INALCO CREE-CEB EA 4513 